\title{
Os fatores de risco e seus mecanismos na gênese do câncer gástrico
}

\author{
Risk factors and their mechanisms in the gastric cancer genesis
}

Factores de riesgo y sus mecanismos en la génesis del cáncer gástrico

Fernanda Rayanny Lourenço Leite ${ }^{1 *}$, Yasmin Lucena Dantas ${ }^{1}$, Marianne Ribeiro Barboza Gaudêncio ${ }^{1}$, Nayara Sayonara Duarte Delgado ${ }^{1}$, Laís Toscano Viana Batista ${ }^{1}$, Klenia Felix de Oliveira Bezerra².

\section{RESUMO}

Objetivo: Analisar os fatores de risco na gênese do câncer gástrico, publicados nos últimos 10 anos. Métodos: Foi realizada uma revisão integrativa da literatura com publicações entre 2008 e 2018 na Biblioteca Virtual em Saúde, indexados nas bases de dados: Literatura Latino-Americana e do Caribe em Ciências da Saúde (LILACS), Medical Literature Analysis and Retrieval System Online (MEDLINE) e Índice Bibliográfico Espanhol de Ciências da Saúde (IBECS). Resultados: Foram identificadas 20 publicações, cujas análises textuais permitiram a construção de duas abordagens temáticas: hábitos e costumes; hábitos dietéticos como fatores de risco para desenvolvimento do câncer gástrico. Vários fatores considerados modificáveis são identificados no processo da carcinogênese gástrica, entre eles destacam-se: o tabagismo, o consumo abusivo de álcool, a obesidade, a ingestão elevada de sódio, o sedentarismo, a baixa ingestão de vegetais e frutas e alta ingestão de carne vermelha. Considerações Finais: Os hábitos, costumes e a ingesta dietética possuem uma relação estreita na carcinogênese gástrica, contribuindo de maneira negativa ou positiva no desenvolvimento e prognóstico dessa malignidade.

Palavras-chave: Câncer gástrico, Fatores de risco, Estilo de vida.

\section{ABSTRACT}

Objective: To analyse the scientific production about the influence of risk factors on the genesis of gastric cancer, published in the last 10 years. Methods: An integrative literature review was carried out with publications between 2008 and 2018 in the Virtual Health Library, indexed in the databases: Latin American and Caribbean Literature in Health Sciences (LILACS), Medical Literature Analysis and Retrieval System Online (MEDLINE) and Spanish Bibliographic Index of Health Sciences (IBECS). Results: 20 publications were identified, textual analyses allowed the construction of two thematic approaches: habits and customs; dietary habits as risk factors for the development of gastric cancer. Several factors considered modifiable are identified in the process of gastric carcinogenesis, among which stand out: smoking, alcohol abuse, obesity, high sodium intake, physical inactivity, low intake of vegetables and fruits and high intake of red meat. Conclusion: Habits, lifestyle and a dietary intake are closely related in gastric carcinogenesis, contributing negatively or positively to the development and prognosis of this malignancy.

Keywords: Gastric cancer, Risk factors, Lifestyle.

\section{RESUMEN}

Objetivo: Analizar la producción científica sobre la influencia de los factores de riesgo en la génesis del cáncer gástrico, publicada en los últimos 10 años. Metodos: Se realizó una revisión integradora de la literatura con publicaciones entre 2008 y 2018 en la Biblioteca Virtual en Salud, indexadas en las bases de datos: Literatura Latinoamericana y Caribeña en Ciencias de la Salud (LILACS), Sistema de Análisis y Recuperación de Literatura Médica en Línea (MEDLINE) y el Índice Bibliográfico Español de Ciencias de la Salud (IBECS).

${ }^{1}$ Faculdade de Ciências Médicas da Paraíba, Cabedelo - PB.

2 Universidade Federal da Paraíba, João Pessoa - PB. *E-mail: fernanadarayanny2009@gmail.com 
Resultados: Se identificaron 20 publicaciones, cuyo análisis textual permitió la construcción de dos enfoques temáticos: hábitos y estilo de vida; los hábitos alimentarios como factores de riesgo para el desarrollo de cáncer gástrico. En el proceso de carcinogénesis gástrica se identifican varios factores considerados modificables, entre ellos: tabaquismo, abuso de alcohol, obesidad, alto consumo de sodio, inactividad física, bajo consumo de verduras y frutas y alto consumo de carnes rojas. Conclusión: Los hábitos, el estilo de vida y la ingesta dietética tienen una estrecha relación en la carcinogénesis gástrica, contribuyendo negativa 0 positivamente al desarrollo y pronóstico de esta malignidad.

Palabras clave: Cáncer gástrico, Factores de riesgo, Estilo de vida.

\section{INTRODUÇÃO}

O câncer gástrico (CG) é a quinta neoplasia mais comum e a terceira maior causa de morte por câncer no mundo. O tipo histológico mais comum, com cerca de $90 \%$ dos casos, é o Adenocarcinoma (RAMPAZZO A, et al., 2012).

Linfomas gástricos, sarcomas, tumores neuroendócrinos e outros mais raros têm potenciais evolutivos e tratamentos diferentes. O câncer do estômago tem prognóstico e tratamento definidos pela localização e estadiamento do tumor e número de linfonodos ressecados e acometidos. Em diversos estudos evidencia-se que mais de $50 \%$ dos pacientes com câncer inicial podem ser curados quando totalmente ressecados (ZILBERSTEIN B, et al., 2013).

O pico de incidência para o câncer de estômago se dá predominantemente em homens, por volta dos 6070 anos. No Brasil, para o triênio 2020-2022, esses tumores aparecerão como o segundo mais frequente na região Norte na incidência entre homens e, em quinto nas regiões Sul e Norte, entre as mulheres. Estima-se que 21.230 novos casos sejam notificados, onde 13.360 serão em homens e 7.870, em mulheres, o que confere um risco estimado de 12,81 a cada 100 mil homens e 7,34 para cada 100 mil mulheres (INCA, 2019).

Vários fatores de risco são identificados como condicionantes para o câncer gástrico em múltiplas populações, mas o seu principal fator é a infecção pela bactéria Helicobacter Pylori. Os fatores de risco externos incluem tabagismo, consumo abusivo de álcool, obesidade, ingestão elevada de sódio e de carne vermelha, baixa atividade física, baixa ingestão de vegetais e frutas e algumas exposições ocupacionais. Existem ainda os fatores de risco hereditários, a exemplo do câncer hereditário difuso gástrico, o adenocarcinoma gástrico e a polipose proximal do estômago, mas, acredita-se que pessoas com estilo de vida saudável teriam menor risco para o desenvolvimento deste tipo de câncer (INCA, 2019; WANG Z, et al., 2017).

O diagnóstico do câncer gástrico é feito a partir da histopatologia, através de biópsia, mas na maioria dos casos, a doença é diagnosticada tardiamente. Tal fato ocorre em decorrência da confusão dos seus sintomas com outras doenças, o que acaba comprometendo o prognóstico (VALLE TD, et al., 2017).

Assim, uma boa forma de estabelecer o prognóstico dessa neoplasia se faz através do estadiamento, que também determinam as estratégias terapêuticas mais adequadas. Os tumores que invadem apenas as camadas mucosa e submucosa ( $\mathrm{T} 1$ ), classificam-se como carcinoma gástrico precoce (CGP). Essas apresentações possuem uma melhor sobrevida, em cinco anos mais de $93 \%$, quando submetidos a cirurgia, dos quais $72,8 \%$. Quando a lesão invade a camada muscular própria (T2), são classificados como câncer gástrico avançado (CGA), e possuem uma taxa de sobrevida inferior (JUCÁ PC, et al., 2012).

Diante desse panorama, com o aumento significativo na incidência do câncer de estômago, da sua prevalência no país, do seu prognóstico e do alto potencial de morte é de extrema importância a investigação acerca dos fatores predisponentes e protetores em sua gênese, uma vez que em sua maioria são fatores modificáveis. Logo, o objetivo do presente estudo buscou analisar a produção científica acerca da influência dos fatores de risco na gênese do câncer gástrico, publicados entre 2008 e 2018. 


\section{MÉTODOS}

Trata-se de uma revisão integrativa de literatura dos últimos 10 anos que partiu da seguinte questão norteadora: Qual o papel dos fatores de risco na gênese do câncer gástrico encontrados nas publicações científicas entre 2008 e $2018 ?$

A busca por fontes bibliográficas ocorreu entre 2018-2019, na Biblioteca Virtual em Saúde (BVS). Utilizouse o cruzamento de descritores indexados no banco de Descritores em Ciência da Saúde (DeCs), interligados pelo operador booleano AND (câncer gástrico AND fatores de risco AND estilo de vida), cujo resultado revelou inicialmente 121 fontes.

Em seguida, apenas 52 fontes foram agrupadas de acordo com os respectivos critérios de inclusão: artigo com texto completo disponível online, publicados entre janeiro de 2008 e dezembro de 2018, indexados na Literatura Latino-Americana e do Caribe em Ciências da Saúde (LILACS), Medical Literature Analysis and Retrieval System Online (MEDLINE) e Índice Bibliográfico Espanhol de Ciências da Saúde (IBECS) com o limite de humanos, nos idiomas português, inglês e espanhol.

A seleção da amostra baseou-se na leitura atenciosa de todos os títulos e resumos e na conformidade dos limites dos objetivos do presente estudo, desconsiderou-se aqueles que, apesar de aparecerem no resultado da busca primária, não abordavam o assunto sob o ponto de vista da pesquisa. Nesse sentido foram excluídos: 10 artigos, por não utilizar o estilo de vida como fator de risco, um artigo em duplicata e 21 artigos por não estarem com texto completo.

Na última etapa, procedeu-se a tabulação das informações em dois quadros de variáveis, organizados no Microsoft Word por: o primeiro referente aos periódicos e ano das publicações, e o segundo referente ao título da obra, autoria, base de dados, eixo temático e tipo de estudo para facilitar a análise e síntese desta revisão.

\section{RESULTADOS}

Das etapas do processo de busca, foram eleitos 20 artigos. Entre as publicações selecionadas, observouse que em 2013 concentrou-se o maior número de artigos, com cinco (25\%) produções, seguidos de 2013 e 2015, com o equivalente a 15\%, cada. 2011, 2014, 2016 e 2017 com duas (10\%) publicações cada, e 2011 com apenas uma (5\%) amostra. Todas as publicações possuem o inglês como idioma principal e a grande maioria (95\%), concentrou-se na base de dados da MEDLINE.

Em relação aos periódicos de publicação, observou-se que o The World Journal of Gastroenterology apresentou o percentual mais expressivo de produções acerca do tema abordado, representando $30 \%$ dos estudos. Esse periódico trata de questões relacionadas a distúrbio de todo o sistema gastrointestinal, fator que explica o maior número de publicações encontradas nele.

O British Journal of Cancer, que é uma das revistas mais citadas quando se analisa avanços sobre o câncer na área de prevenção, diagnóstico e tratamento, ficou representada com $15 \%$ das publicações, seguida pela International Journal of Cancer, que correspondeu a $10 \%$ da amostra. Vale destacar que as revistas foram divididas conforme os anos de publicações (Quadro 1).

Conforme o eixo temático, $75 \%$ das publicações abordaram os Hábitos dietéticos em seus estudos, e $45 \%$ os Hábitos e costumes, sendo observados publicações que retratavam de ambos os eixos, $20 \%$. Já em relação ao tipo de estudo, o mais encontrado foi o estudo de coorte, totalizando $50 \%$ do corpo amostral, seguido do caso-controle com $25 \%$, meta-análise com $10 \%$, ecológico, ensaio clínico e revisão sistemática, cada um compondo $5 \%$ das publicações. O corpo amostral segundo o título, autoria, base de dados, eixo temático e tipo de estudo também foram agrupados (Quadro 2). 


\section{Revista Eletrônica Acervo Saúde / Electronic Journal Collection Health | ISSN 2178-2091}

Quadro 1 - Distribuição dos artigos publicados por periódico e número de publicações por ano no período de 2008 a 2018.

\begin{tabular}{|c|c|c|c|c|c|c|c|c|c|c|c|c|}
\hline Periódicos & 2008 & 2009 & 2010 & 2011 & 2012 & 2013 & 2014 & 2015 & 2016 & 2017 & 2018 & $\mathrm{~N}$ \\
\hline $\begin{array}{c}\text { The American Journal of } \\
\text { Clinical Nutrition }\end{array}$ & - & - & 1 & - & - & - & - & - & - & - & - & 1 \\
\hline $\begin{array}{c}\text { The World Journal of } \\
\text { Gastroenterology }\end{array}$ & - & - & - & 1 & - & 2 & 2 & 1 & - & - & - & 6 \\
\hline Annals of Epidemiology & - & - & - & 1 & - & - & - & - & - & - & - & 1 \\
\hline British Journal of Cancer & - & - & - & - & 1 & - & - & 1 & - & 1 & - & 3 \\
\hline Cancer Causes Control & - & - & - & - & - & 1 & - & - & - & - & - & 1 \\
\hline $\begin{array}{l}\text { International Journal of } \\
\text { Cancer }\end{array}$ & - & - & - & - & 1 & - & - & - & 1 & - & - & 2 \\
\hline $\begin{array}{l}\text { The American Journal of } \\
\text { Clinical Nutrition }\end{array}$ & - & - & - & - & 1 & - & - & - & - & - & - & 1 \\
\hline The Journal of Nutrition & - & - & - & - & - & 1 & - & - & - & - & - & 1 \\
\hline Plos one & - & - & - & - & - & 1 & - & - & - & - & - & 1 \\
\hline Nutrición Hospitalaria & - & - & - & - & - & - & - & 1 & - & - & - & 1 \\
\hline Cambridge Core & - & - & - & - & - & - & - & - & 1 & - & - & 1 \\
\hline Journal of Epidemiology & - & - & - & - & - & - & - & - & - & 1 & - & 1 \\
\hline Total & 0 & 0 & 1 & 2 & 3 & 5 & 2 & 3 & 2 & 2 & 0 & 20 \\
\hline
\end{tabular}

Fonte: Leite FRL, et al., 2020. 


\section{Revista Eletrônica Acervo Saúde / Electronic Journal Collection Health | ISSN 2178-2091}

Quadro 2 - Distribuição dos artigos publicados conforme: título, autoria, base de dados, eixo temático e tipo de estudo.

\begin{tabular}{|c|c|c|c|c|}
\hline Título & Autoria & Base de dados & Eixo & Tipo de estudo \\
\hline $\begin{array}{l}\text { Gastric cancer and salt preference: a population- } \\
\text { based cohort study in Korea }\end{array}$ & KIM J, et al., 2010. & MEDLINE & Hábitos dietéticos; & Estudo de coorte \\
\hline $\begin{array}{l}\text { A case-control study on the relationship between salt } \\
\text { intake and salty taste and risk of gastric cancer }\end{array}$ & YANG WG, et al., 2011. & MEDLINE & Hábitos dietéticos; & Caso-controle \\
\hline $\begin{array}{l}\text { Principal component analysis of dietary and lifestyle } \\
\text { patterns in relation to risk of subtypes of esophageal } \\
\text { and gastric cancer }\end{array}$ & SILVERA SAN, et al., 2011. & MEDLINE & Hábitos dietéticos; & Caso-controle \\
\hline $\begin{array}{c}\text { Prediagnosis lifestyle exposures and survival of } \\
\text { gastric câncer patients: a cohort study from Portugal }\end{array}$ & FERRONHA I, et al., 2012. & MEDLINE & Hábitos e costumes; & Estudo de coorte \\
\hline $\begin{array}{l}\text { Fruit and vegetable intake and the risk of gastric } \\
\text { adenocarcinoma: A reanalysis of the european } \\
\text { prospective investigation into câncer and nutrition } \\
\text { (EPIC-EURGAST) study after a longer follow-up }\end{array}$ & GONZÁLEZ CA, et al., 2012. & MEDLINE & Hábitos e costumes; & Estudo de coorte \\
\hline $\begin{array}{l}\text { Dietary flavonoid and lignan intake and gastric } \\
\text { adenocarcinoma risk in the European Prospective } \\
\text { Investigation into Cancerand Nutrition (EPIC) study }\end{array}$ & ZAMORA-ROS R, et al., 2012. & MEDLINE & $\begin{array}{l}\text { Hábitos dietéticos; } \\
\text { Hábitos e costumes; }\end{array}$ & Estudo de coorte \\
\hline $\begin{array}{c}\text { Risk factors for gastric } \\
\text { cancer in Latin America: a meta-analysis. }\end{array}$ & BONEQUI P, et al., 2013. & MEDLINE & $\begin{array}{l}\text { Hábitos dietéticos; } \\
\text { Hábitos e costumes; }\end{array}$ & Meta-análise \\
\hline $\begin{array}{c}\text { Plasma Isoflavone Concentrations Are Not } \\
\text { Associated with Gastric Cancer Risk among } \\
\text { Japanese Menand Women }\end{array}$ & HARA A, et al., 2013. & MEDLINE & Hábitos dietéticos; & Caso-controle \\
\hline $\begin{array}{l}\text { Evaluation of the } \\
\text { relationship between dietary factors, CagA-positive } \\
\text { Helicobacter pylori infection, and RUNX3 } \\
\text { promoter hypermethylation in gastric cancer tissue. }\end{array}$ & ZHANG YW, et al., 2013. & MEDLINE & Hábitos dietéticos; & Caso-controle \\
\hline $\begin{array}{l}\text { Study of risk factors for gastric cancer by } \\
\text { populational data bases analysis }\end{array}$ & $\begin{array}{l}\text { FERRARI F e REIS MAM, } \\
\qquad 2013 .\end{array}$ & MEDLINE & Hábitos dietéticos; & Ecológico \\
\hline
\end{tabular}


Revista Eletrônica Acervo Saúde / Electronic Journal Collection Health | ISSN 2178-2091

\begin{tabular}{|c|c|c|c|c|}
\hline Título & Autoria & Base de dados & Eixo & Tipo de estudo \\
\hline $\begin{array}{c}\text { Physical Activity and Sedentary Behavior in Relation } \\
\text { to Esophageal and Gastric Cancers in the NIH-AARP } \\
\text { Cohort }\end{array}$ & COOK MB, et al., 2013. & MEDLINE & Hábitos e costumes; & Estudo de coorte \\
\hline $\begin{array}{l}\text { Dairy product consumption and gastric cancer risk: A } \\
\text { meta-analysis }\end{array}$ & SUN Y, et al., 2014. & MEDLINE & Hábitos dietéticos; & Meta-análise \\
\hline $\begin{array}{c}\text { Gene-diet interactions in gastric cancer risk: A } \\
\text { systematic review }\end{array}$ & KIM J, et al., 2014 & MEDLINE & Hábitos dietéticos; & Revisão sistemática \\
\hline Diet and Upper Gastrointestinal Malignancies & ABNET CC, et al., 2015. & MEDLINE & $\begin{array}{l}\text { Hábitos dietéticos; } \\
\text { Hábitos e costumes; }\end{array}$ & Ensaio clínico \\
\hline $\begin{array}{c}\text { Impact of alcohol drinking on gastric cancer } \\
\text { development according to Helicobacter pylori } \\
\text { infection status }\end{array}$ & MA SH, et al., 2015. & MEDLINE & Hábitos e costumes; & Estudo coorte \\
\hline $\begin{array}{c}\text { Risk and protective factors for gastric metaplasia and } \\
\text { cancer: a hospital-based case-control study in } \\
\text { Ecuador }\end{array}$ & SALVADOR I, et al., 2015 & IBECS & Hábitos dietéticos; & Caso-controle \\
\hline $\begin{array}{c}\text { Worldwide burden of gastric cancer in } 2012 \text { that } \\
\text { could have been prevented by increasing fruit and } \\
\text { vegetable intake and predictions for } 2025\end{array}$ & PELETEIRO B, et al., 2016. & MEDLINE & Hábitos dietéticos; & Estudo de coorte \\
\hline $\begin{array}{l}\text { Fruit and vegetable consumption, Helicobacter pylori } \\
\text { antibodies, and gastric cancer risk: A pooled analysis } \\
\text { of prospective studies in China, Japan, and Korea. }\end{array}$ & WANG T, et al., 2016. & MEDLINE & Hábitos dietéticos; & Estudo de coorte \\
\hline $\begin{array}{c}\text { Demographic and lifestyle factors and survival } \\
\text { among patients with esophageal and gastric cancer: } \\
\text { The Biobank Japan Project. }\end{array}$ & OKADA E, et al., 2017. & MEDLINE & Hábitos e costumes; & Estudo de coorte \\
\hline $\begin{array}{c}\text { Composite protective lifestyle factors and risk of } \\
\text { developing gastric adenocarcinoma: the Singapore } \\
\text { Chinese Health Study. }\end{array}$ & WANG Z, et al., 2017 & MEDLINE & $\begin{array}{l}\text { Hábitos dietéticos; } \\
\text { Hábitos e costumes; }\end{array}$ & Estudo de coorte \\
\hline
\end{tabular}

Fonte: LEITE FRL, et al., 2020.

REAS/EJCH | Vol.13(2) | e5627 | DOI: https://doi.org/10.25248/reas.e5627.2021 Página 6 de 11


Na etapa seguinte, os artigos foram organizados de acordo com a equivalência temática, em dois eixos: 1 - Maus hábitos como fatores de risco para o câncer gástrico; 2 - Dieta como fator de risco para o câncer gástrico.

\section{Maus hábitos como fatores de risco para o câncer gástrico}

No que concerne ao enfoque abordado nesta categoria, $75 \%$ dos artigos deram ênfase aos vários fatores considerados modificáveis no processo da carcinogênese gástrica, entre eles destacam-se: o tabagismo, o consumo abusivo de álcool, a obesidade, a ingestão elevada de sódio, o sedentarismo, a baixa ingestão de vegetais e frutas e alta ingestão de carne vermelha. Esses fatores de risco geralmente ocorrem em conjunto, agravando a situação. Em contrapartida, a manutenção de um estilo de vida saudável possui um menor risco no desenvolvimento dessa malignidade (YANG WG, et al., 2011). No entanto, Zamora-Ros R, et al. (2012) não identificam os bons hábitos de vida como fatores protetivos.

No estudo realizado por Bonequi $P$, et al. (2013) observou-se que há uma forte associação entre o tabagismo e o câncer gástrico. Fumantes atuais possuem uma chance de aproximadamente $60 \%$ maior, quando comparado aos que nunca fumaram. Tal situação ocorre porque os carcinógenos do tabaco tanto agridem diretamente a mucosa gástrica como também favorecem a persistência do H. pylori, diminuindo a eficácia da terapia de erradicação.

Em uma análise mais recente realizada pela Agência Internacional de Pesquisa sobre o Câncer (IARC), em 2017, apontou o tabagismo como carcinógeno do grupo I, ou seja, altamente potencial. Um cigarro, além de conter mais de 70 substâncias cancerígenas conhecidas, incluindo as nitrosaminas específicas do tabaco e hidrocarbonetos aromáticos policíclicos, possui também alto nível de nicotina. Sabe-se que a nicotina é responsável por ativar os receptores nicotínicos de acetilcolina e induzir a proliferação celular nas linhagens de câncer gástrico, regulando positivamente a ciclooxigenase (WANG Z, et al., 2017).

Em contraste, Ferronha I, et al. (2012) demonstrou que o efeito do tabagismo depende do tipo histológico e estágio do tumor, sugerindo um risco aumentado de morte para os pacientes que fumam mais de 20 maços/ano. Os fumantes possuem concentrações sanguíneas mais baixas de ácido ascórbico e carotenóides, pois a nicotina favorece o estresse oxidativo dessas substâncias, eliminando-as mais facilmente. A vitamina C é um importante aliado para evitar a carcinogênese gástrica, visto que ela atua inibindo a formação endógena de nitrosaminas e combate os radicais livres oxidativos, que são potencialmente mutagênicos (GONZÁLEZ CA, et al., 2012; WANG Z, et al., 2017).

Em relação ao consumo de álcool, os seus mecanismos na gênese do câncer gástrico ainda não estão totalmente elucidados. Alguns estudos sugerem que o álcool causa tanto danos mecânicos diretos quanto 0 cigarro, visto que aumenta a secreção ácida e a gerando de espécies reativas de oxigénio e radicais livre, induzindo um ambiente inflamatório nesse órgão. Já os danos indiretos estão relacionados a mudanças alimentares, principalmente em alcoólatras, e são dependentes da dose consumida (BONEQUI P, et al. 2013; MA SH, et al., 2015). Segundo Ma SH, et al. (2015) há uma associação significativa com o consumo pesado de álcool ( $\geq 4$ doses por dia) e a chance de desenvolver câncer gástrico, no entanto não há associação com o consumo moderado de álcool.

A associação entre o consumo de álcool e o risco de câncer é complexa, sabe-se que as bebidas alcoólicas são metabolizadas em acetaldeído, um metabólito potencialmente carcinogênico, classe 1 pelo IARC. Desse modo, quanto maior o teor alcoólico de uma bebida, mais danosa essa será para o organismo (ABNET CC, et al., 2015).

Em 2015, um estudo de coorte prospectivo, multicêntrico, coreano investigou a associação entre padrões de consumo de álcool e o risco de câncer gástrico em uma população de 18.863 participantes. Investigou-se que havia uma associação estatisticamente significativa quando da longa duração do consumo de álcool e, observou-se que a frequência do consumo de sete ou mais vezes por semana, em torno de $55 \mathrm{~g}$ de álcool. Foram associadas com 3,48 vezes e 3,27 vezes, respectivamente, com maior risco para o câncer gástrico, em comparação com os não-etilistas infectados por H. pylori (MA SH, et al., 2015). 
O sedentarismo, o sobrepeso e a obesidade também são apontados como fatores de risco para o câncer gástrico. Um estudo de coorte realizado em 2013 revelou uma associação positiva entre o elevado índice de massa corporal (IMC) e essa malignidade. Porém não conseguiu estabelecer soberania na prevenção desse câncer por pessoas que praticam atividades físicas constantemente daquelas que são sedentárias (COOK MB, et al., 2013).

Já no estudo de Okada E, et al. (2017) observaram que pacientes com câncer gástrico, que possuem baixo peso, apresentam uma pior sobrevida do que aqueles que possuem um IMC elevado, ou seja, pacientes desnutridos, com falta de nutrientes e queda do estado imunológico têm pior prognóstico para o câncer gástrico quando comparados a obesos.

Wang Z, et al. (2017) revelou que o sobrepeso e a obesidade (IMC>25) estão significativamente associados a um maior risco de desenvolver câncer gástrico e um pior prognóstico. Isso ocorre devido presença de citocinas pró-inflamatórias produzidas pelo excesso de gordura corporal que pode levar à inflamação crônica. $O$ excesso de gordura corporal pode também supra regular o fator de crescimento semelhante à insulina-1, que estimula a proliferação celular e inibe a apoptose.

\section{Dieta como fator de risco para o câncer gástrico.}

No tocante à dieta, os estudos publicados permitiram conhecer a influência deste fator na carcinogênese. $\mathrm{Na}$ última década, as pesquisas publicadas têm propiciado um maior conhecimento sobre o papel da dieta na gênese do câncer gástrico. Tem sido sugerido que propriedades antioxidantes, frutas e vegetais potencialmente melhoram os efeitos do $\mathrm{H}$. pylori protegendo o epitélio gástrico da resposta inflamatória e prevenindo a nitrosação endógena, evento carginogênico (WANG T, et al., 2016).

Acredita-se que a prevenção desse câncer pode ser realizada com o aumento na ingestão de frutas e vegetais em porções de até 300 e $400 \mathrm{~g} / \mathrm{d}$, respectivamente. O "Projeto GBD (Global Burden of Disease)" analisou, em 161 países, o número absoluto de casos de câncer que poderiam ser evitados com um aumento na ingestão de frutas. Essa associação reduziria em 4.900 casos o número de neoplasias malignas do estômago (PELETEIRO B, et al., 2016).

Uma alimentação equilibrada, com frutas e verduras possuem efeitos quimiopreventivos, advindos dos altos níveis de micronutrientes (incluindo antioxidantes), o que pode diminuir o dano ao DNA, que causaria uma diminuição dos telômeros, pela eliminação de radicais livres de oxigênio. O comprimento dos telômeros está associado ao estresse oxidativo cumulativo de fatores ambientais, estando mais curtos em pessoas com câncer gástrico (ABNET CC, et al., 2015).

Ferrari $F$, et al. (2013) analisaram a associação entre a incidência de câncer gástrico e a exposição populacional a fatores de risco e de proteção, por meio da análise de bancos de dados internacionais. Foi identificado que maiores valores de índice de desenvolvimento humano (IDH) são associados a uma maior prevalência de obesidade masculina e um maior consumo per capita de álcool, tabaco, e carne, e que também estão associados a uma maior incidência de câncer gástrico. Em contraste, um alto consumo de vegetais foi associado a uma menor incidência de doenças em outros países.

Zamora-Ros R, et al. (2012) encontraram uma associação inversa significativa entre o consumo total de flavonoides e o risco de câncer gástrico em mulheres, mas não em homens. Os flavonóides são compostos fenólicos que ocorrem amplamente em alimentos de origem vegetal, como frutas e legumes, chá, vinho e produtos de cacau. In vitro, altas doses de flavonóides mostraram fortes propriedades anticarcinogênicas, particularmente devido à sua capacidade de modular o metabolismo de carcinógenos, regulando vias inflamatórias, inibindo a proliferação celular e induzindo a apoptose.

Bonequi $P$, et al. (2013) corroboram com essa afirmação visto que evidenciam que 0 alto consumo de frutas e verduras possuem um efeito gastroprotetor, contribuindo com a defesa antioxidante do organismo, evitando os danos oxidativos em macromoléculas. Segundo Abnet CC, et al. (2015), as flavonas, presentes em frutas e hortaliças também possuem efeitos quimiopreventivos, inibindo o processo celular associado à carcinogênese: adesão, invasão e migração. 
Os resultados da pesquisa de Zhang YW, et al. (2013) sugerem ainda que vários fatores dietéticos, incluindo folato, metionina e vitaminas $\mathrm{B} 12$ e B6, foram relacionados com o risco de câncer observadas em estudos epidemiológicos devido a seu papel na metilação do DNA. A alta ingestão de folato e vitaminas B6 e B12 podem proteger contra essa metilação do gene RUNX3, que é um candidato a gene supressor de tumor e que, acredita-se, desempenha um papel na progressão do câncer gástrico.

Já o consumo de carne vermelha ou processada tem sido associado ao aumento do risco de câncer gastrointestinal devido ao alto teor de aminas heterocíclicas, compostos $\mathrm{N}$-nitrosos e hidrocarbonetos aromáticos policíclicos gerados durante métodos de cocção específicos, bem como o alto teor de ferro-heme da carne vermelha. Para piorar, quando a carne é submetida ao processamento, possuem um acréscimo no teor do nitrato. Essas substâncias podem ser tóxicas e são capazes de reagir com o DNA do organismo, alterando suas bases e iniciam o processo da carcinogênese (ABNET CC, et al., 2015). Para Wang Z, et al. (2017) o maior nível de ingestão de ferro heme, presente na carne vermelha foi associado a um aumento significativo de $67 \%$ no risco de adenocarcinoma gástrico.

Um estudo multicêntrico observou uma associação positiva entre o risco de adenocarcinomas do esôfago e da cárdia gástrica e consumo de carne e proteína animal, visto que apresentou evidência de uma relação inversa com a ingestão de fibras dietéticas. Para outros adenocarcinomas gástricos, sugere-se uma associação positiva com alimentos contendo nitrito nos países ocidentais e alimentos salgados ou conservados nos países asiáticos (SILVERA SAN, et al., 2011). De acordo com Hara A, et al. (2013) as isoflavonas, substâncias encontradas na soja e seus derivados, não foi associada ao desenvolvimento de câncer gástrico.

No estudo de Bonequi $P$, et al. (2013) foi elucidado que o consumo de carne vermelha e carne processada, principalmente, com sal em excesso, aumenta o risco de câncer gástrico. Abnet CC, et al. (2015) acreditam que existem vários mecanismos pelos quais o sal pode contribuir para a carcinogênese gástrica. $A$ ingestão de sal aumenta as chances de desenvolver gastrite, e aumenta os efeitos dos carcinógenos gástricos, como a N-metil- N-nitro- $\mathrm{N}$-nitrosoguanidina. Além disso, altas concentrações salinas no estômago podem romper a barreira mucosa e levar à inflamação e atrofia. Para Wang Z, et al. (2017) a administração de alta concentração de cloreto de sódio na dieta causa dano imediato à mucosa gástrica, aumenta a proliferação celular e altera a viscosidade da mucosa.

O sal irrita a mucosa no estômago resultando em dano celular, fator que aumenta o risco de câncer por potencializar a ação de carcinógenos e aumento na possibilidade de mutações endógenas. O seu consumo em altas doses foi associado a alterações atróficas na mucosa gástrica, aumentando o risco de desenvolver câncer gástrico posteriormente (KIM J, et al., 2010). Além disso, Yang WG, et al. (2011) verificaram em seu estudo de caso controle que pacientes com câncer gástrico possuem uma pontuação mais elevada STST, um teste útil para avaliar a preferência do sabor salgado e ingestão de sal, logo são mais propensos a ter preferência sabor salgado e uma alta ingestão de alimentos salgados.

Salvador I, et al. (2015) realizaram um estudo de caso-controle baseado em hospitais conduzido no Equador, no qual verificou que a adição de sal a mais de $50 \%$ dos alimentos é fator de risco significativamente associado à presença de câncer gástrico e metaplasia. Propõe-se que o papel causal da ingestão de sal no desenvolvimento de Câncer deve-se à maior proliferação do epitélio gástrico no corpo e antro proximais, bem como uma diminuição no número de células parietais, e ao fato de que o sal danifica diretamente a mucosa do estômago de maneira dose-dependente (KIM J, et al., 2010).

Em relação ao consumo de leite e derivados, uma meta-análise avaliou trinta e oito publicações com estudos de caso-controle e estudos de coorte que exploraram uma possível associação entre o consumo de produtos lácteos e o risco de câncer gástrico. Embora haja a afirmação de que o consumo frequente e a longo prazo de produtos lácteos pode levar à obesidade que aumenta as respostas imunes pró-inflamatórias e acelera a carcinogênese gástrica induzida pelo Helicobacter, o consumo de produtos lácteos foi associado a um aumento não significativo do risco de câncer gástrico, sendo necessárias novas investigações (SUN Y, et al., 2014). 
O consumo de chá parece ter efeito protetor contra a carcinogênese gástrica, uma vez que o seu consumo regular esteve associado à diminuição do risco de desenvolvimento do câncer gástrico. Este resultado sugere que o efeito protetor de seu consumo contra esse tipo de câncer é independente dos mecanismos de desintoxicação de carcinógenos químicos exógenos (KIM J, et al., 2014).

O modo de preparo dos alimentos também pode estar relacionado ao risco para o câncer gástrico. $O$ consumo de alimentos reaquecidos pelo menos 3 vezes por semana representa um importante fator de risco. Esse reaquecimento das refeições expõe repetidamente a comida temperaturas elevadas prolongadas que podem levar à formação de acrilamida, aminas heterocíclicas e policíclicos hidrocarbonetos aromáticos, que são compostos mutagênicos formados durante exposição a alta temperatura de carnes (SALVADOR I, et al., 2015).

\section{CONSIDERAÇÕES FINAIS}

A análise das fontes elegíveis permitiu concluir que é de suma importância o conhecimento dos fatores de risco relacionados ao estilo de vida e seus mecanismos no processo de carcinogênese gástrica, que em sua maioria podem ser modificados. O estudo apontou que existe uma relação estreita entre os maus hábitos e costumes, bem como a ingestão de alguns tipos de alimentos com o desenvolvimento e o pior prognóstico do câncer gástrico. Fatores de risco modificáveis como o uso excessivo do álcool, do tabagismo e a obesidade foram considerados precursores desse tipo de câncer. Já a prática regular de atividade física, foi apontada como um efeito protetor. A dieta também apresentou um papel condicionante na gênese do câncer de estômago. Destacou-se, no estudo, o efeito quimiopreventivo do consumo frequente de frutas e verduras, devido aos nutrientes e substâncias presentes em sua composição. A ingestão de chá também foi associada com a diminuição do risco no desenvolvimento de câncer gástrico. Já o consumo de carnes vermelhas, processadas, alimentos reaquecidos, e principalmente a ingestão de sal, apresentaram efeito potencialmente carcinogênico. É indispensável a manutenção de bons hábitos e da prática de um estilo de vida mais saudável, com a finalidade de prevenir o surgimento da doença e melhorar a proposta terapêutica e o prognóstico nesse tipo de malignidade.

\section{REFERÊNCIAS}

1. ABNET CC, et al. Diet and Upper Gastrointestinal Malignancies. Gastroenterology, 2015; 148(6): 1234-1243.e4.

2. BONEQUI $P$, et al. Risk factors for gastric cancer in Latin America: a meta-analysis. Cancer Causes Control, 2013; 24(2): 217-231.

3. COOK MB, et al. Physical Activity and Sedentary Behavior in Relation to Esophageal and Gastric Cancers in the NIHAARP Cohort. PLoS ONE, 2013; 8(12): e84805.

4. FERRARI F, REIS MAM. Study of risk factors for gastric cancer by populational databases analysis. World $\mathrm{J}$ Gastroenterol, 2013; 19(48): 9383-9391.

5. FERRONHA I, et al. Prediagnosis lifestyle exposures and survival of gastric cancer patients: a cohort study from Portugal. British Journal of Cancer, 2012; 107(3): 537-543.

6. GONZÁLEZ CA, et al. Fruit and vegetable intake and the risk of gastric adenocarcinoma: A reanalysis of the european prospective investigation into cancer and nutrition (EPIC-EURGAST) studyafter a longer follow-up. International Journal of Cancer [S.L.], 2012; 131(12): 2910-2919.

7. HARA A, et al. Plasma Isoflavone Concentrations Are Not Associated with Gastric Cancer Risk among Japanese Men and Women. The Journal Of Nutrition, 2013; 143(8): 1293-1298.

8. INCA - Instituto Nacional de Câncer José Alencar Gomes da Silva. Coordenação de Prevenção e Vigilância. Estimativa de Câncer 2020: Incidência de câncer no Brasil. Rio de Janeiro (RJ), 2019.

9. JUCÁ PC, et al. Comparação da sobrevivência e dos fatores prognósticos em pacientes com adenocarcinoma gástrico T2 e T3. Revista do Colégio Brasileiro de Cirurgiões, 2012; 39(5): 377-384.

10. KIM J, et al. Gene-diet interactions in gastriccancerrisk: A systematic review. World Journal of Gastroenterology, 2014; 20(28): 9600-9610.

11. KIM J, et al. Gastric cancer and salt preference: a population-based cohort study in Korea. The American Journal of Clinical Nutrition, 2010; 91(5):1289-93.

12. $\mathrm{MA} \mathrm{SH}$, et al. Impact of alcohol drinking on gastric cancer development according to Helicobacter pylori infection status. British Journal of Cancer, 2015; 113(9):1381-8.

13. OKADA E, et al. BioBank Japan Cooperative Hospital Group, Tamakoshi A. Demographic and lifestyle factors and survival among patients with esophageal and gastric cancer: The Biobank Japan Project. Journal of Epidemiology 2017; 27(3S): S29-S35. 
14. PELETEIRO B, et al. Worldwide burden of gastric cancer in 2012 that could have been prevented by increasing fruit and vegetable intake and predictions for 2025. British Journal of Nutrition, 2016;115(5):851-9.

15. RAMPAZZO A, et al. Gastric adenocarcinoma trends in the central region of Rio Grande do Sul (Southern Brazil): what has changed in 25 years?. Arq. Gastroenterol, 2012; 49(3): 178-183.

16. SALVADOR I, et al. Risk and protective factors for gastric metaplasia and cancer: a hospital-based case-control study in Ecuador. Nutr. Hosp, 2015; 32(3):1193-9.

17. SILVERA SAN, et al. Principal Component Analysis of Dietary and Lifestyle Patterns in Relation to Risk of Subtypes of Esophageal and Gastric Cancer. Annals of Epidemiology, 2011;21(7):543-50.

18. SUN Y, et al. Dairy product consumption and gastric cancer risk: A meta-analysis. World Journal of Gastroenterology, 2014;20(42):15879-98.

19. VALLE TD, et al. Intervening factors for the initiation of treatment of patients with stomach and colorectal cancer. Rev. Latino-Am. Enfermagem, 2017; 25(1): e2879.

20. WANG T, et al. Fruit and vegetable consumption, Helicobacter pylori antibodies, and gastric cancer risk: A pooled analysis of prospective studies in China, Japan, and Korea. International Journal of Cancer, 2016;140(3):591-599.

21. WANG Z, et al. Composite protective lifestyle factors and risk of developing gastric adenocarcinoma: the Singapore Chinese Health Study. British Journal of Cancer, 2017;116(5):679-687.

22. YANG WG, et al. A case-control study on the relationship between salt intake and salty taste and risk of gastric cancer. World Journal of Gastroenterology, 2011;17(15):2049-53.

23. ZAMORA-ROS R, et al. Dietary flavonoid and lignan intake and gastric adenocarcinoma risk in the European Prospective Investigation into Cancer and Nutrition (EPIC) study. The American Journal of Clinical Nutrition, 2012; 96(6): 1398-408.

24. ZHANG YW, et al. Evaluation of the relationship between dietary factors, Cag A-positive Helicobacter pylori infection, and RUNX3 promoter hypermethylation in gastric cancer tissue. World Journal of Gastroenterology, 2013; 19(11): 1778-87.

25. ZILBERSTEIN B, et al. J, Brazilian consensus in gastric cancer: guidelines for gastric cancer in Brazil. ABCD Arq Bras Cir Dig ,2013; 26(1): 2-6. 